\title{
Tumor-directed gene therapy in mice using a composite nonviral gene delivery system consisting of the piggyBac transposon and polyethylenimine
}

\author{
Yu Kang1,2, Xiaoyan Zhang1,2, Wei Jiang1,2, Chaoqun $\mathrm{Wu}^{3}$, Chunmei Chen ${ }^{3}$, \\ Yufang Zheng ${ }^{4}$, Jianren $\mathrm{Gu}^{5}$ and Congjian $\mathrm{Xu}^{* 1,2,6}$
}

\begin{abstract}
Address: ${ }^{1}$ Obstetrics and Gynecology Hospital, Fudan University, Shanghai 200011, PR China, ${ }^{2}$ Department of Obstetrics and Gynecology of Shanghai Medical School, Fudan University, Shanghai 200011, PR China, ${ }^{3}$ The State Key Laboratory of Genetic Engineering, School of Life Sciences, Fudan University, Shanghai 200433, PR China, ${ }^{4}$ Department of Biophysics, School of Life Sciences, Fudan University, Shanghai 200433, PR China, ${ }^{5}$ National Laboratory of Oncogene and Related Genes, Shanghai Cancer Institute, Shanghai 200032, PR China and ${ }^{6}$ Institutes of Biomedical Sciences, Fudan University, Shanghai 200032, PR China

Email: Yu Kang - yukang@fudan.edu.cn; Xiaoyan Zhang - xyzh03@163.com; Wei Jiang - jw52317@hotmail.com; ChaoqunWu - cqwu@fudan.edu.cn; Chunmei Chen - cmchen@fudan.edu.cn; Yufang Zheng - zhengyf@fudan.edu.cn; Jianren Gu -nlorg@sh163.net; CongjianXu* - xucj@hotmail.com

* Corresponding author
\end{abstract}

Published: 27 April 2009

BMC Cancer 2009, 9:126 doi:10.1186/1471-2407-9-126

This article is available from: http://www.biomedcentral.com/147/-2407/9/126

(C) 2009 Kang et al; licensee BioMed Central Ltd.

This is an Open Access article distributed under the terms of the Creative Commons Attribution License (http://creativecommons.org/licenses/by/2.0), which permits unrestricted use, distribution, and reproduction in any medium, provided the original work is properly cited.
Received: 30 September 2008

Accepted: 27 April 2009

\begin{abstract}
Background: Compared with viral vectors, nonviral vectors are less immunogenic, more stable, safer and easier to replication for application in cancer gene therapy. However, nonviral gene delivery system has not been extensively used because of the low transfection efficiency and the short transgene expression, especially in vivo. It is desirable to develop a nonviral gene delivery system that can support stable genomic integration and persistent gene expression in vivo. Here, we used a composite nonviral gene delivery system consisting of the piggyBac (PB) transposon and polyethylenimine (PEI) for long-term transgene expression in mouse ovarian tumors.
\end{abstract}

Methods: A recombinant plasmid PB [Act-RFP, HSV-tk] encoding both the herpes simplex thymidine kinase (HSV-tk) and the monomeric red fluorescent protein (mRFPI) under PB transposon elements was constructed. This plasmid and the PBase plasmid were injected into ovarian cancer tumor xenografts in mice by in vivo PEl system. The antitumor effects of HSV-tk/ ganciclovir (GCV) system were observed after intraperitoneal injection of GCV. Histological analysis and TUNEL assay were performed on the cryostat sections of the tumor tissue.

Results: Plasmid construction was confirmed by PCR analysis combined with restrictive enzyme digestion. mRFPI expression could be visualized three weeks after the last transfection of $\mathrm{pPB} / \mathrm{TK}$ under fluorescence microscopy. After GCV admission, the tumor volume of PB/TK group was significantly reduced and the tumor inhibitory rate was $81.96 \%$ contrasted against the $43.07 \%$ in the TK group. Histological analysis showed that there were extensive necrosis and lymphocytes infiltration in the tumor tissue of the PB/TK group but limited in the tissue of control group. TUNEL assays suggested that the transfected cells were undergoing apoptosis after GCV admission in vivo.

Conclusion: Our results show that the nonviral gene delivery system coupling PB transposon with PEI can be used as an efficient tool for gene therapy in ovarian cancer. 


\section{Background}

Gene therapy is a promising strategy for the treatment of unresectable cancer. Non-viral gene transfer systems used in cancer gene therapy are attractive because they are relatively stable, safer, and easier to produce than viral vectors [1]. Conventional nonviral gene transfer systems include the direct tissue injection of DNA or transfection across the cell membrane using liposomes, peptide delivery systems, or polymer vectors[2]. Among them, the polycation polyethylenimine (PEI) have been extensively used for in vivo gene delivery [3]. PEI combines strong DNA compaction capacity with an intrinsic endosomolytic activity[4]. Recently studies showed that PEI derivatives can mediate efficient gene delivery in several tumors, such as lymphoma [5], hepatocellular carcinoma [6], pancreatic tumors [7], and ovarian carcinoma [8]. However, a significant drawback to PEI-mediated gene transfer delivery is that there still is no persistent transgene expression as there is no stable chromosomal integration [9]. The development of nonviral gene-transfer technologies that can support stable chromosomal integration and persistent gene expression in vivo is desirable.

Transposon system is a major step in overcoming that barrier as it is a technology that combines the advantages of nonviral delivery with genomic integration and persistent transgene expression. Transposon-derived sequences account for more than $40 \%$ of the genome in humans and mice $[10,11]$, indicating the importance of transposition in evolution. Their ability to deliver and integrate specific genetic cargo into a host's chromosome has made transposons so useful as a molecular genetic tool in invertebrate species, and now they has progressively become more important as delivery vectors of therapeutic expression cassettes to treat human disease[12,13]. The main advantages of transposon-mediated genetic cargo delivery are: 1) Transgene in a chromosomally integrated transposon can be persistent expressed; 2) Transposon lacks viral sequences that could elicit unwanted immune or inflammatory responses [14]; 3) Some transposons have the capacity to accommodate large size foreign genes $[15,16]$.

One of the well characterized DNA transposon vector used in mammals is the synthetic Sleeping Beauty (SB) transposon system. So far, SB transposon has been used successfully to mediate stable gene transfer and expression in several mouse and human cells [17-19]. However, as a transgene tool for therapeutic gene delivery in mammals, SB transposons was limited due to the facts that exhibits overproduction inhibition which limits in vivo activity [20] and that had a limited capacity to carry DNA fragments[21]. Recently, several other transposon vectors have been tested for their potential to deliver therapeutic genes, including Tol2[22], Frog Prince[23], and piggy$\operatorname{Bac}(\mathrm{PB})[15,24-27]$. These vectors differ in their efficiency of gene insertion, genetic cargo capacity, integration site preferences, and effects on chromosomal stability. Among them, PB, a functional DNA transposon from cabbage looper moth Trichoplusia ni, now was the widely used one in human gene therapy and mammalian mutagenesis. Wu et al. found that PB is the most active transposons compared with SB, Tol2 and Mos1. They also showed that PB lacks of overproduction inhibition, which is advantageous in preclinical development of transposon-based gene therapy[26].

Moreover, Ding and coworkers designed a binary cotransfection assay system consisting of both a donor plasmid containing the transposon and a helper plasmid containing the transposase. The donor plasmid, named PB [ActRFP] DS, can carry multiple genes, which allows one to perform complex transgenic experiments such as identifying positive transgenic animals with the help of a visible marker. Their studies showed that cotransfection of donor plasmid and helper plasmid produced drug-resistant clones on average 50-fold higher than donor plasmid transfection alone, which confirmed that the enhanced clone production was due to trans-position. They suggested that the PB transposon can accommodate large foreign genes and readily mediate the introduction of foreign genes up to $14 \mathrm{~kb}$ in length. Therefore it can be an efficient transgenesis and insertional mutagenesis tool in mice and other vertebrate organisms[15].

In the present work, we adopted this PB transposon system carrying the herpes simplex thymidine kinase gene (HSV-tk gene) and delivered it to ovarian tumor grafted mice using PEI as the transfection reagent. The therapeutic potential of this nonviral gene delivery system was evaluated in an established mouse model of ovarian cancer. This is the first demonstration that PB combined with PEI as nonviral gene transfer system can be used as an efficient transgene tool for gene therapy in ovarian cancer.

\section{Methods}

\section{Tumor cell lines}

The cell line SKOV3 established from a human ovarian adenocarcinoma was obtained from the Shanghai Institute of Cell Biology of Chinese Academy of Sciences (Shanghai, China). Cells were grown in McCoy's 5A medium supplemented with $10 \%(\mathrm{~V} / \mathrm{V})$ fetal bovine sera, $100 \mathrm{U} / \mathrm{mL}$ of penicillin, and $100 \mathrm{mg} / \mathrm{mL}$ streptomycin (all purchased from GIBCO BRL, Grand Island, New York, USA). Cells were maintained at $37^{\circ} \mathrm{C}$ in a humidified incubator containing $5 \% \mathrm{CO}_{2}$ and cultures were split twice per week.

\section{Plasmid construction}

The plasmid PB [Act-RFP]DS (pPB), containing the mRFP1 expression cassette, and plasmid ActPBase(pPBase), containing PB transposase were kindly provided by Xiaohui Wu (Institute of Developmental 
A

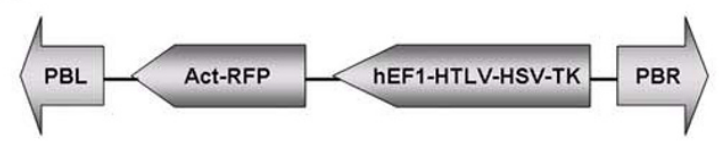

PB[Act-RFP,HSV-TK]

C

$\begin{array}{lllll}M & 1 & 2 & 3 & 4\end{array}$

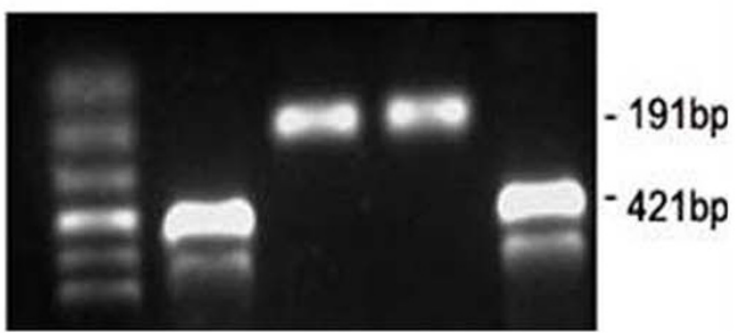

B

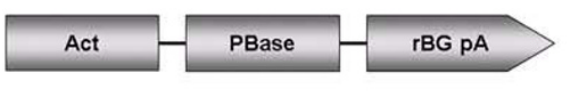

Act-PBase

D

$\begin{array}{llll}M & 1 & 2 & 3\end{array}$

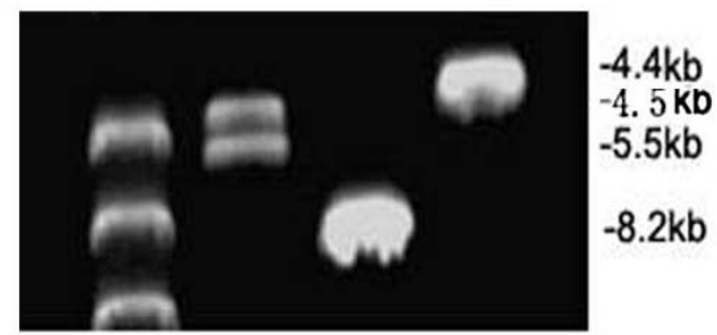

\section{Figure I}

Construction of recombinant Plasmid PB [Act-RFP, HSV-tk]. (A) PB [Act-RFP, HSV-tk] constructs. The HSV-tk gene was cloned in the BamHI-Spel sites of the PB [Act-RFP] DS (pPB), generating PB [Act-RFP, HSV-tk] (pPB/TK), which consists of both HSV-tk and mRFPI expression cassettes. PBL and PBR was a pair of PB repeat termini; (B) PB transposase helper constructs. The piggyBac transposase gene (PBase) driven by $\beta$-actin (Act) promoters was followed by rabbit $\beta$-globin polyA (rBG PA) (adapted from Ref [I5]); (C) PCR analysis of PPB/TK: Lane I: Marker; Lane 2: PPB/TK: PCR product of tk fragment; Lane 3: pPB/TK: PCR product of mRFPI fragment; Lane 4: pPB: PCR product of mRFPI fragment; Lane 5: pORF-HSVtk: PCR product of tk fragment; (D) Restriction analysis of pPB/TK: Lane I: Marker; Lane 2: pPB/TK; Lane 3: pPB; Lane 4: pORF-HSVtk.

Biology and Molecular Medicine, School of Life Sciences, Fudan University, Shanghai, China). The plasmid pORFHSVtk encoding the HSV-tk gene was obtained from Invivogen (San Diego, CA, USA). To generate the recombinant plasmid PB [Act-RFP, HSV-TK], named as $\mathrm{PPB} / \mathrm{TK}$, we first amplified HSV-tk cDNA using the forward primer 5'-ATTGGATCCGCTCCGGTGCCCGTCAGTGG-3' and the reverse primer 5'-CTAACTAGTTCAGTTAGCCTCCCCCATCTCC-3'. The PCR fragment was TA cloned into pMD18-T Simple vector (Takara, Dalian, China) and subcloned into BamHI-SpeI sites of PB [Act-RFP] DS. Plasmid construction was identified by PCR analysis combined with restriction enzyme digestion. The primer sequences for HSV-tk were: forward primer, 5'-CCTGTGGTGCCTCCTGAACT-3'; reverse primer, 5'-GTTGCTATGGCCGCGAGAAC- 3 '. The predicted product size is 421 bp. For mRFP1, the forward primer was 5'-GGACGGCGAGTTCATCTACA-3', and the reverse primer was 5'-TTGACCTCGGCGTCGTAGTG-3', and the predicted product size is $191 \mathrm{bp}$.

\section{Tumor model}

Animal study in this research has been performed with the approval of Animal Ethics Committee of Obstetrics and Gynecology Hospital, Fudan University. Female nude mice BALB/ $c$ at 6 weeks old were purchased from the Animal Center of the Chinese Academy of Science, Shanghai, China, and maintained in specific pathogen-free facility. All procedures were performed in accordance with The Guide for the Ethical Treatment of Laboratory Animals from the Ministry of Science and Technology of People's Republic of China (Publication No. 2006-398). To generate ovarian cancer tumor xenografts, SKOV3 cells $\left(1 \times 10^{7}\right.$ cells $/ 0.1 \mathrm{ml}$ of medium/mouse) were inoculated subcutaneously in the right flank of mice. Animals were inspected and weighed every three days.

\section{Complex formation and administration}

In vivo-jetPEI was obtained from Polyplus-transfection (Illkirch, France). PEI/DNA complexes were prepared as follows: $3.2 \mu \mathrm{l}$ of $150 \mathrm{mM}$ PEI derivative in $5 \%$ glucose solution were vortex mixed with $10 \mu \mathrm{g}$ of pPB/TK DNA and $10 \mu \mathrm{g}$ of plasmid Act-PBase DNA in a final volume of $100 \mu \mathrm{l}$ of $5 \%$ glucose solution. The glucosylated PEI/DNA mixture $(\mathrm{N} / \mathrm{P}$ ratio $=8)$ was left for 15 minutes at room temperature, and then injected into xenografted mice intratumorally.

\section{Gene delivery to tumor in vivo}

Transfection experiments were performed two weeks after tumor inoculation. Twenty mice were assigned to four groups: $\mathrm{PB} / \mathrm{TK}$ treatment group (transfected with $\mathrm{pPB} / \mathrm{TK}$ 

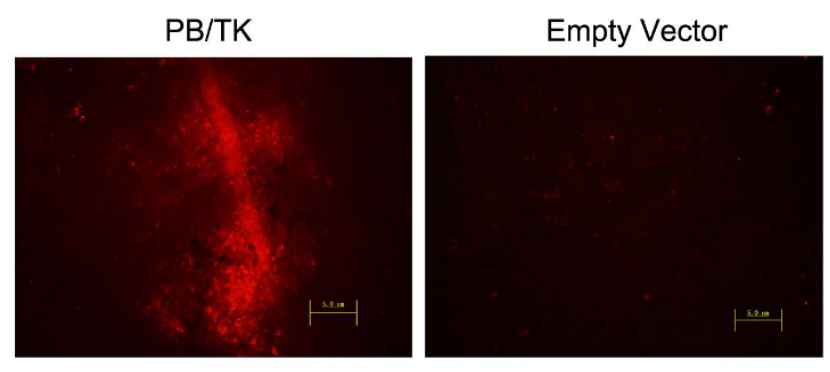

Figure 2

Transgene expression in vivo. Fluorescence micrographs of PPB/TK-transfected tumor cryosections show that mRFPI expression was observed three weeks after transfection. Empty vector was used as control. (Magnification, $\times 200$ ).

and pPBase), PB group (transfected with $\mathrm{pPB}$ and pPBase), TK group (transfected with pORF-HSVtk) and control group ( $5 \%$ (vol/vol) dextrose). Each group contained an equal number of large and intermediate-sized tumors so that the mean tumor volume in each group was comparable before intratumoral injection. Every mouse was intratumorally injected with $100 \mu \mathrm{l}$ PEI/DNA complexes once a day on days 1,4 and 7. Twenty-four hours after the first transfection, $25 \mathrm{mg} / \mathrm{kg}$ ganciclovir (GCV) was injected intraperitoneally twice daily for 7 days. Tumor size was measured with calipers two times per week, and tumor volume was estimated from two-dimensional tumor measurements using the formula: $\mathrm{V}=$ length

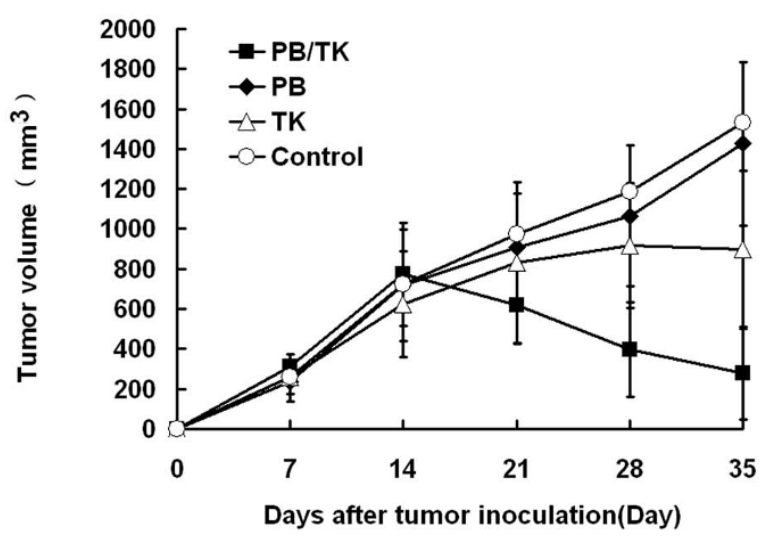

Figure 3

Tumor growth inhibition assay. Two weeks after subcutaneous inoculation of SKOV3 cells in BALB/c nude mice, complexes of pPB/TK, pPB, pORF-HSVtk with PEl were injected directly into the tumor three times (days I, 4 and 7). GCV solution was administered by daily intraperitoneal injection beginning on the day after the first complex injections. Tumor volumes were monitored over time. Mice were sacrificed 3 weeks after transfection in vivo. Errors bar represent the standard error of the mean.* $p<0.01$, comparing the PB/ TK group with the control group at day $2 \mathrm{I}$. $(\mathrm{mm}) \times$ width $^{2}\left(\mathrm{~mm}^{2}\right) / 2$. Three weeks after the last transfection in vivo, mice were euthanized and necropsies were performed. The weights of tumors were measured to determine the tumor weight $(\mathrm{g})$ and inhibitory rate $(\%)$. The tumor inhibitory rate was calculated using the following formula: Inhibitory rate $=[$ (tumor weight of control group-tumor weight of experiment group)/tumor weight of control group] $\times 100 \%$. After measuring final tumor weight, all tumors and major organs, such as heart, lung, liver, spleen, kidney, were fixed in formalin and embedded in paraffin for further sections. Histopathological analysis was performed following routine hematoxylin and eosin (H\&E) staining on sections.

\section{Assaying transgene expression}

To monitor transgene expression, the mRFP1 reporter gene was assessed in frozen tumor sections. Three weeks after the last transfection of $\mathrm{pPB} / \mathrm{TK}$ and intraperitoneal injection of GCV, mice were scarified and formaldehydefixed $6-\mu \mathrm{m}$ frozen sections were rinsed in PBS and mounted on glass slides. mRFP1 expression was visualized using fluorescence microscopy (Nikon Eclipse E600, Tokyo, Japan). Empty vector pPB combined with PEI was used as control.

\section{Immunofluorescence costaining}

To examine the apoptosis of transfected cells, which was induced by GCV in vivo, cryostat sections of tumors were fixed with acetone and analyzed for TUNEL-positive cells using a fluorescence in situ cell death detection kit (Roche Molecular Biochemicals, Indianapolis, IN) according to the manufacturer's instructions. In brief, sections were treated with the TUNEL reaction mixture (including FITCconjugated dUTP) and incubated in the dark for $1.5 \mathrm{~h}$ at $37^{\circ} \mathrm{C}$, followed by PBS washes. Sections were examined using a fluorescence microscope.

\section{Statistical analysis}

Results are expressed as means \pm SD. One-way ANOVA was used to evaluate differences between groups, and a $p$ value less than 0.05 was considered significant.

\section{Results \\ Recombinant plasmid construction}

The HSV-tk gene was cloned within the BamHI-SpeI sites of the $\mathrm{PB}$ transposon $\mathrm{pPB}$, generating recombinant plasmid $\mathrm{pPB} / \mathrm{TK}(10 \mathrm{~kb})$ containing both mRFP1 and the HSV-tk expression cassettes in Figure 1A. Figure 1B illustrates the helper plasmid which contains a PB transposase gene (PBase) driven by $\beta$-actin (Act) promoters and followed by rabbit $\beta$-globin polyA (rBG pA). Figure $1 \mathrm{C}$ shows positive $\mathrm{pPB} / \mathrm{TK}$ clones confirmed by PCR amplification of the HSV-tk and mRFP1 genes fragments at 421 bp and $191 \mathrm{bp}$, respectively. We also confirmed the positive clone by restriction digest with HindIII. The correct 
Table I: Tumor weight on the 2 I st day after transfection in vivo

\begin{tabular}{lll}
\hline Group & Weight of tumor $(\mathbf{X} \pm \mathbf{S D}, \mathbf{g})$ & Tumor inhibitory rate $(\mathbf{w} / \mathbf{w}, \%)$ \\
\hline PB/TK & $0.31 \pm 0.23$ & $81.96 *$ \\
PB & $1.61 \pm 0.41$ & 6.40 \\
TK & $0.98 \pm 0.36$ & 43.07 \\
Control & $1.72 \pm 0.54$ & \\
\hline
\end{tabular}

Growth inhibition was observed in the PB/TK group compared with the other groups. ${ }^{*} p<0.01$, comparing the PB/TK group with the control group.

clones of $\mathrm{pPB} / \mathrm{TK}$ generated two fragments at $5.5 \mathrm{~kb}$ and $4.5 \mathrm{~kb}$, while digestion of pPB or pORF-HSVtk yielded a single band at $8.2 \mathrm{~kb}$ and $4.4 \mathrm{~kb}$, respectively (Figure $1 \mathrm{D}$ ).

\section{Transposition mediates transgene expression in vivo} As mRFP1 is a marker for transgene expression, so mRFP1 expression in tumors was examined to ascertain whether $\mathrm{PB}$ transposon-mediated gene transfer supports stable gene expression in mice. The results indicated that the mRFP1 expression could be observed even three weeks after transfection on $\mathrm{pPB} / \mathrm{TK}$-transfected tumor cryosections, but not in empty vector controls (Figure 2).

\section{Tumor growth inhibition after HSV-TK gene transfer and GCV administration}

To test the in vivo sensitivity of the $\mathrm{pPB} / \mathrm{TK}$ transfected cells to GCV, the mice were cotransfected with $\mathrm{pPB} / \mathrm{TK}$ and pPBase using PEI, and subsequently treated with GCV. Figure 3 shows that the tumor volume of $\mathrm{PB} / \mathrm{TK}$ group was

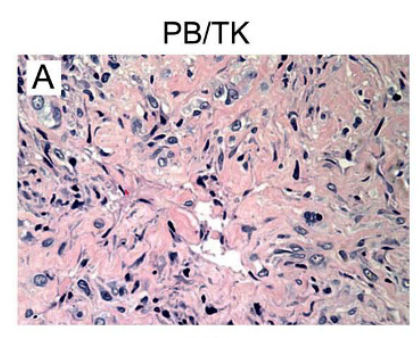

PB

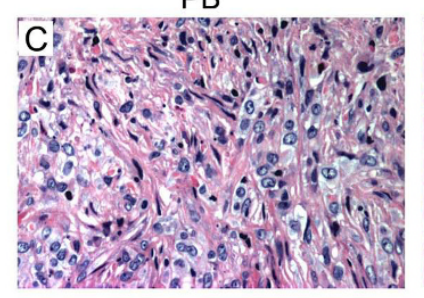

\section{Figure 4}

H\&E staining of tumor tissue samples removed from different subgroups. PB/TK group: Extensive necrosis; TK group: Minimal necrosis but moderate lymphocyte aggregation around tumor cells; PB group \& Control group: Enlarged hyperchromatic and pleomorphic nuclei with irregular nuclear membrane and nucleoli. (Magnification, $\times 200$ ). significantly reduced, while much less tumor volume was observed in the TK group. At day 21, the tumor inhibitory rate in $\mathrm{PB} / \mathrm{TK}$ group was $81.96 \%$ contrast to $43.07 \%$ in the TK group ( $p<0.01$, Table 1$)$. No difference in the rate was observed between the PB group and control group. In all animals, no noticeable toxicity was observed as no obvious damage on major organs was discovered by histopathological analysis (data not shown). The results demonstrate that nonviral gene transfer by $\mathrm{PB}$ transposon combined with PEI might result in potent tumor growth inhibition.

\section{Histological Analysis of different subgroups}

To determine the antitumor efficacy of the HSV-tk/GCV system in vivo, paraffin-embedded tissues were sectioned at $4 \mu \mathrm{m}$ and stained with $\mathrm{H} \& \mathrm{E}$. The sections from the tumor tissue of the $\mathrm{PB} / \mathrm{TK}$ group showed extensive necrosis and lymphocyte infiltration, as shown in figure $4 \mathrm{~A}$. Meanwhile, minimal necrosis and moderate lymphocyte infiltration were identified in the TK group sections (Figure 4B). Necrosis was absent in tumor samples from both the $\mathrm{PB}$ and control groups (Figure 4C, D).

\section{In vivo killing of transfected cells by GCV}

To evaluate the killing mechanism of transfected cells treated with GCV, TUNEL assays were performed on mouse tumor sections. Transfected cells were shown as bright red with mRFP1 expression (Figure 5A), and apoptotic cells were labeled with bright green (Figure $5 \mathrm{~B}$ ). The merged image (yellow) suggested that almost all transfected cells were undergoing apoptosis after GCV treatment in vivo (Figure 5C).

\section{Discussion}

Ovarian carcinoma is a common cancer worldwide, and there is still no effective therapy for such unresectable disease. Gene therapy may represent an attractive alternative to the classical treatments, and numerous experimental approaches have shown potential promise in treatment of ovarian carcinoma [28-30]. Among them, several protocols are based on the use of suicide genes such as the HSVtk gene, which can induce significant reduction in tumor burden and can prolong survival after the prodrug GCV treatment. However, the clinical application of this 

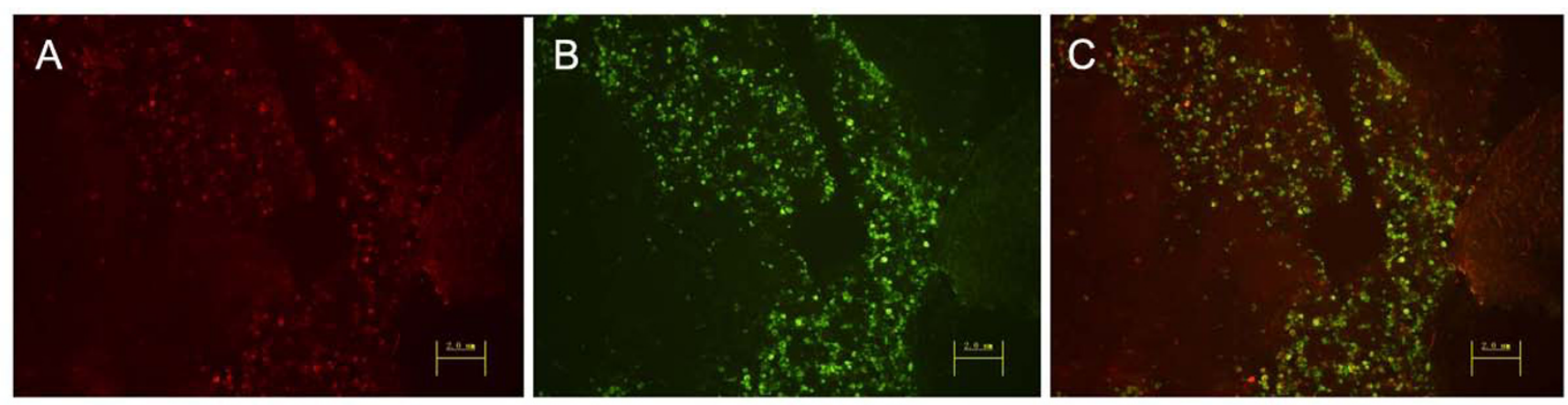

Figure 5

In vivo sensitivity of the HSV-tk transfected cells to GCV (Magnification, $\mathbf{\times 2 0 0}$ ). (A) The PB/TK transfected cells are shown as bright red. (B) Apoptotic cells are bright green after staining with TUNEL assay kit. (C) The merged cells (yellow) suggest the transfected cells were undergoing apoptosis after GCV treatment in vivo.

approach may be hindered by transgene vectors. Current viral vectors carry a risk for generating active viral particles through recombination, and triggering unwanted host inflammatory and immune responses $[31,32]$, while nonviral vectors lack the ability to induce stable chromosomal integration and persistent gene expression in vivo [33].

In recent years there has been an increased understanding of the mechanisms behind transgene delivery. Successful delivery of foreign genes to a mammalian cell involves several discrete steps: delivery of genes to target cells, cellular entry, endosome escape, and integration into the nucleus $[34,35]$. To date, no single gene transfer system has been developed that can effectively fulfill the requirements for all of the features described above. A combination of complementary, multi-functional nonviral gene transfer systems may satisfy the major requirements for efficient gene delivery.

Cationic gene carriers have a net positive charge and can facilitate the cellular entry of DNA by interacting with the negatively charged cell surface $[35,36]$. Endosomal escape is achieved by including components that induce membrane fusion at low $\mathrm{pH}$ or block $\mathrm{pH}$ lowering inside the endosome in the DNA-carrier complex. PEI is a cationic polymer that serves as an effective vehicle for in vivo gene delivery in many tissues. It facilitates effective DNA binding and protection, combined with the capability to escape the endosome due to its proton buffering ability with resultant osmotic swelling and endosomal disruption [36]. PEI has been shown to be an efficient transfection agent for ovarian carcinoma cells by Intraperitoneal injection [8]. However, several studies indicated that PEImediated transgene expression was dose-dependent and transient, with maximal transgene expression observed during the 48-hour period immediately following transfection [37]. Very low amounts of DNA remained detectable after $72 \mathrm{~h}$, indicating probable degradation and clearance [8]. Thus, the development of nonviral gene- transfer technologies that can support stable chromosomal integration and persistent gene expression in vivo is desirable.

Transposon-mediated DNA delivery has opened the door to the development of a new generation of vectors for human gene therapy and mammalian [12]. Transposonbased systems have been proven to be effective integrating nonviral vectors that can mediate long-term in vivo transgene expression [17]. Ohlfest and coworkers used SB transposons as an intratumoral gene transfer vector for glioblastoma multiforme, and delivered using PEI as gene transfer reagent. They observed marked anti-tumor activity as demonstrated by reduced tumor vessel density, inhibition of tumor growth, and tumor elimination in up to $50 \%$ of nude mice[38,39]. In the present study, we used $\mathrm{PB}$, a more active transposon[15,24-27], combined with PEI to deliver HSV-TK gene into ovarian cancer xenografts. Our results demonstrate that the expression of foreign DNA mediated by PB transposon and PEI is much more efficient than PEI alone. After HSV-TK gene transfer and GCV administration, significant tumor growth inhibition in vivo was observed up to $82 \%$. The tumor inhibition rate is much higher than previous study by SB mediated gene therapy, probably due to PB transposon is more efficient than SB transposon. Long-term expression and bystander effect of HSV-TK gene may contribute to the result. However, we still do not know the exact mechanism how PB transposon could cause more tumor inhibition effect in vivo. Further studies should be considered to determine how $\mathrm{PB}$ transposon performs in vivo in long term studies. Furthermore, for PB transposon therapy to transition into clinical consideration, the potential safety issues from insertional disruption or deregulation in genome will also need to be addressed.

\section{Conclusion}

In summary, our results demonstrate the feasibility and effectiveness of a nonviral gene transfer system combind- 
ing the PB transposon with PEI. This system has shown great efficiency on treating ovarian caner in our model, however, the therapeutic effect of our method against other carcinoma needs further investigation.

\section{Abbreviations}

PB transposon: piggyBac transposon; PEI: polyethylenimine; HSV-tk: herpes simplex thymidine kinase; mRFP1: monomeric red fluorescent protein; GCV: ganciclovir; TUNEL: terminal deoxynucleotidyl transferase dUTP nick end labeling; SB transposon: Sleeping Beauty transposon; PCR: polymerase chain reaction.

\section{Competing interests}

The authors declare that they have no competing interests.

\section{Authors' contributions}

YK helped design some of the experiments, performed the in vitro and in vivo studies and drafted the manuscript; $\mathrm{XYZ}$ and WJ carried out the animal studies; CQW and CMC provided technical assistance throughout the study and analyzed the data; JRG and YFZ helped design the experiment and participated in completing manuscript. CJX conceived the study, and participated in its design and coordination. All authors read and approved the final manuscript.

\section{Acknowledgements}

The authors thank the Institute of Developmental Biology and Molecular Medicine, Fudan University for providing piggyBac reagents generated under the support of the Chinese National Basic Research Program (973) Grant 2006CB806700 and Chinese National High-tech Research and Development Program (863) Grant 2007AA022 100. We are grateful to Long Yu (The State Key Laboratory of Genetic Engineering, School of Life Sciences, Fudan University, Shanghai, China) for providing experimental facilities and helpful suggestions. This study was supported by grants from the Research Scholar Foundation of Fudan University, China (NO: 06L-Y02), the National Natural Sciences Foundation of China (No: 30700902), the Shanghai Health Bureau Youth Projects (NO: 2006Y04), Shanghai Rising-Star Program (NO: 08QA I 4016), the Shanghai Leading Academic Discipline Project (NO: BI I7), and the Chinese National High-tech Research and Development Program (863) (NO: 2006AA02Z342).

\section{References}

I. Ohlfest JR, Freese AB, Largaespada DA: Nonviral vectors for cancer gene therapy: prospects for integrating vectors and combination therapies. Current gene therapy 2005, 5(6):629-64I.

2. Hughes RM: Strategies for cancer gene therapy. Journal of surgical oncology 2004, 85(I):28-35.

3. Nimesh S, Chandra R: Guanidinium-grafted polyethylenimine: an efficient transfecting agent for mammalian cells. Eur J Pharm Biopharm 2008, 68(3):647-655.

4. Godbey WT, Wu KK, Mikos AG: Tracking the intracellular path of poly(ethylenimine)/DNA complexes for gene delivery. Proceedings of the National Academy of Sciences of the United States of America 1999, 96(9):5177-5181.

5. Weecharangsan W, Opanasopit P, Lee RJ: Effect of depsipeptide on in vitro transfection efficiency of PEI/DNA complexes. Anticancer research 2008, 28(2A):793-798.

6. Iwai M, Harada Y, Tanaka S, Muramatsu A, Mori T, Kashima K, Imanishi J, Mazda O: Polyethylenimine-mediated suicide gene transfer induces a therapeutic effect for hepatocellular car- cinoma in vivo by using an Epstein-Barr virus-based plasmid vector. Biochem Biophys Res Commun 2002, 29 I ( I):48-54.

7. Vernejoul F, Faure P, Benali N, Calise D, Tiraby G, Pradayrol L, Susini $C$, Buscail L: Antitumor effect of in vivo somatostatin receptor subtype 2 gene transfer in primary and metastatic pancreatic cancer models. Cancer research 2002, 62(2I):6I24-6I 31 .

8. Louis MH, Dutoit S, Denoux Y, Erbacher P, Deslandes E, Behr JP, Gauduchon P, Poulain L: Intraperitoneal linear polyethylenimine (L-PEI)-mediated gene delivery to ovarian carcinoma nodes in mice. Cancer gene therapy 2006, I3(4):367-374.

9. Romano G: Current development of nonviral-mediated gene transfer. Drug news \& perspectives 2007, 20(4):227-23I.

10. Lander ES, Linton LM, Birren B, Nusbaum C, Zody MC, Baldwin J, Devon K, Dewar K, Doyle M, FitzHugh W, et al.: Initial sequencing and analysis of the human genome. Nature 200I, 409(6822):860-921.

II. Waterston RH, Lindblad-Toh K, Birney E, Rogers J, Abril JF, Agarwal P, Agarwala R, Ainscough R, Alexandersson M, An P, et al.: Initial sequencing and comparative analysis of the mouse genome. Nature 2002, 420(69 I 5):520-562.

12. Hackett PB, Ekker SC, Largaespada DA, Mclvor RS: Sleeping beauty transposon-mediated gene therapy for prolonged expression. Advances in genetics 2005, 54:189-232

13. Miskey C, Izsvak Z, Kawakami K, Ivics Z: DNA transposons in vertebrate functional genomics. Cell Mol Life Sci 2005, 62(6):629-64l.

14. Marshall E: Gene therapy death prompts review of adenovirus vector. Science 1999, 286(5448):2244-2245.

15. Ding S, Wu X, Li G, Han M, Zhuang Y, Xu T: Efficient transposition of the piggyBac (PB) transposon in mammalian cells and mice. Cell 2005, I 22(3):473-483.

16. Hackett CS, Geurts AM, Hackett PB: Predicting preferential DNA vector insertion sites: implications for functional genomics and gene therapy. Genome biology 2007, 8(Suppl I):SI2.

17. Yant SR, Meuse L, Chiu W, Ivics Z, Izsvak Z, Kay MA: Somatic integration and long-term transgene expression in normal and haemophilic mice using a DNA transposon system. Nature genetics 2000, 25(I):35-4I.

18. Belur LR, Podetz-Pedersen K, Frandsen J, Mclvor RS: Lung-directed gene therapy in mice using the nonviral Sleeping Beauty transposon system. Nature protocols 2007, 2(I 2):3|46-3 I 52.

19. Ohlfest JR, Frandsen JL, Fritz S, Lobitz PD, Perkinson SG, Clark KJ, Nelsestuen G, Key NS, Mclvor RS, Hackett PB, et al.: Phenotypic correction and long-term expression of factor VIII in hemophilic mice by immunotolerization and nonviral gene transfer using the Sleeping Beauty transposon system. Blood 2005 , I 05(7):2691-2698.

20. Wilson $M H$, Kaminski JM, George $A L$ Jr: Functional zinc finger/ sleeping beauty transposase chimeras exhibit attenuated overproduction inhibition. FEBS letters 2005 , 579(27):6205-6209.

21. Izsvak Z, Ivics Z, Plasterk RH: Sleeping Beauty, a wide hostrange transposon vector for genetic transformation in vertebrates. Journal of molecular biology 2000, 302(I):93-102.

22. Kawakami K: Tol2: a versatile gene transfer vector in vertebrates. Genome biology 2007, 8(SuppI I):S7.

23. Miskey C, Izsvak Z, Plasterk RH, Ivics Z: The Frog Prince: a reconstructed transposon from Rana pipiens with high transpositional activity in vertebrate cells. Nucleic acids research 2003, 3 I (23):6873-688I.

24. Feschotte C: The piggyBac transposon holds promise for human gene therapy. Proceedings of the National Academy of Sciences of the United States of America 2006, I 03(4I): |498|-|4982.

25. Morales ME, Mann VH, Kines KJ, Gobert GN, Fraser MJ Jr, Kalinna $\mathrm{BH}$, Correnti JM, Pearce EJ, Brindley PJ: piggyBac transposon mediated transgenesis of the human blood fluke, Schistosoma mansoni. Faseb J 2007, 2 I ( I 3):3479-3489.

26. Wilson $\mathrm{MH}$, Coates C], George AL Jr: PiggyBac transposonmediated gene transfer in human cells. Mol Ther 2007, I 5(I): I39-145.

27. Wu SC, Meir YJ, Coates C], Handler AM, Pelczar P, Moisyadi S, Kaminski JM: piggyBac is a flexible and highly active transposon as compared to sleeping beauty, Tol2, and Mos I in mammalian cells. Proceedings of the National Academy of Sciences of the United States of America 2006, I 03(4I): I5008-I50I3. 
28. Agarwal R, Linch M, Kaye SB: Novel therapeutic agents in ovarian cancer. Eur J Surg Oncol 2006, 32(8):875-886.

29. Kimball KJ, Numnum TM, Rocconi RP, Alvarez RD: Gene therapy for ovarian cancer. Current oncology reports 2006, 8(6):44 I-447.

30. Kang Y, Xu CJ, Wu CQ, Liu XS, Zhong CP, Zhang XH, Qiao SY, Gu JR: A novel strategy to compensate the disadvantages of live vaccine using suicide-gene system and provide better antitumor immunity. Vaccine 2006, 24(I 2):2| |4I-2I 50.

31. Baum C, Fehse B: Mutagenesis by retroviral transgene insertion: risk assessment and potential alternatives. Current opinion in molecular therapeutics 2003, 5(5):458-462.

32. Graham A, Walker R, Baird P, Hahn CN, Fazakerley JK: CNS gene therapy applications of the Semliki Forest virus I vector are limited by neurotoxicity. Mol Ther 2006, 13(3):63 I-635.

33. Nishikawa $M$, Huang $L$ : Nonviral vectors in the new millennium: delivery barriers in gene transfer. Human gene therapy 200I, I 2(8):86 |-870.

34. Min SH, Lee DC, Lim MJ, Park HS, Kim DM, Cho CW, Yoon do Y, Yeom YI: A composite gene delivery system consisting of polyethylenimine and an amphipathic peptide KALA. The journal of gene medicine 2006, 8( I 2): I425-I434.

35. Audouy S, Hoekstra D: Cationic lipid-mediated transfection in vitro and in vivo (review). Molecular membrane biology 200I, 18(2):129-143.

36. Lungwitz U, Breunig M, Blunk T, Gopferich A: Polyethyleniminebased non-viral gene delivery systems. Eur J Pharm Biopharm 2005, 60(2):247-266.

37. Dolivet G, Merlin JL, Barberi-Heyob M, Ramacci C, Erbacher P, Parache RM, Behr JP, Guillemin F: In vivo growth inhibitory effect of iterative wild-type $\mathrm{p} 53$ gene transfer in human head and neck carcinoma xenografts using glucosylated polyethylenimine nonviral vector. Cancer gene therapy 2002, 9(8):708-7।4.

38. Ohlfest JR, Demorest ZL, Motooka Y, Vengco I, Oh S, Chen E, Scappaticci FA, Saplis RJ, Ekker SC, Low WC, et al.: Combinatorial antiangiogenic gene therapy by nonviral gene transfer using the sleeping beauty transposon causes tumor regression and improves survival in mice bearing intracranial human glioblastoma. Mol Ther 2005, I 2(5):778-788.

39. Ohlfest JR, Lobitz PD, Perkinson SG, Largaespada DA: Integration and long-term expression in xenografted human glioblastoma cells using a plasmid-based transposon system. Mol Ther 2004, I0(2):260-268.

\section{Pre-publication history}

The pre-publication history for this paper can be accessed here:

http://www.biomedcentral.com/1471-2407/9/126/pre

pub
Publish with Biomed Central and every scientist can read your work free of charge

"BioMed Central will be the most significant development for disseminating the results of biomedical research in our lifetime. "

Sir Paul Nurse, Cancer Research UK

Your research papers will be:

- available free of charge to the entire biomedical community

- peer reviewed and published immediately upon acceptance

- cited in PubMed and archived on PubMed Central

- yours - you keep the copyright
BioMedcentral 